\title{
AN EFFICIENT MODELING OF FLEXIBLE BLIMPS: EULERIAN APPROACH
}

\author{
Selima Bennaceur* \\ Laboratoire IBISC, CNRS FRE 2378, \\ Université d'Evry Val d'Essonne, 40 Rue du Pelvoux, \\ 91020 Evry cedex, France
}

\author{
Naoufel Azouz \\ Laboratoire IBISC, CNRS FRE 2378, \\ Université d'Evry Val d'Essonne, 40 Rue du Pelvoux, \\ 91020 Evry cedex, France
}

\author{
Azgal Abichou \\ Laboratoire d'Ingénieurie Mathématique, \\ Ecole Polytechnique de Tunisie B.P. 743 - 2078 La \\ Marsa, Tunisia
}

\begin{abstract}
Unmanned Aerial Vehicles (U.A.V.) have a need of a greater autonomy in their new missions. Autonomous U.A.V. flight control systems require a precise modeling of the dynamic behavior taking into account the effect of the flexibility and the interaction with the surrounding fluid. In this paper, we present an efficient modeling of the autonomous flexible blimps. These flying objects are assumed to undergo large rigid-body motion and small elastic deformations. The formalism used is based on the Newton-Euler approach. This one is frequently used for flying rigid objects. In this study we develop a method to generalize the existing Newton-Euler "rigid body" formalisms by including the effect of the flexibility without destroying the global methodology. The method is hybrid. It uses the Lagrange equations and the Eulerian variables. The flexibility appears in the global dynamical system by the way of few supplementary degrees of freedom. This method has the advantage of making easier the elaboration of algorithms of control, stabilization or generation of trajectories. The added mass phenomenon is also taken into account in the dynamical system. This phenomenon is important for big and light objects moving in a fluid such as airships. As validation we use the parameters of an AS-200 blimp belonging to the University of Evry.
\end{abstract}

\section{INTRODUCTION}

Autonomous aerial vehicles such as airships and drones have recently gained importance. The airships began these last decades to retrieve a new youth after a half century of hibernation. Capability of airships are expanding rapidly now, and the range of missions they designed to support is growing.
We can give the examples of climate research, surveillance or even infiltration in war scenarios, and more closer to us we always saw them as means of advertising. A common denominator in all these situations is the impossibility or unwillingness to have human presence at the scene. In that case a precise dynamic model should be elaborated to permit an easy control, stabilization or navigation task of this autonomous object. In order to fulfill this requirement, it is necessary to introduce the effect of the structural flexibility in the dynamic model. It is important to note that several kinds of airships, usually called blimps, are mainly constituted of a balloon filled with gas. The only solid parts are the careen and the tail fins (for more details see [1]). The integration of the structural flexibility in the dynamic analysis is then useful; however it is now in an embryonic state and is only just emerging. Several researches were done by using the assumption of rigid body behavior for airships [2],[3]. The flexibility effects are sometimes modeled as a perturbation. However in other flying objects, such as light aircrafts, the introduction of the flexibility in the dynamic model becomes essential $[4,5]$. Thus we try to contribute to the study of the deformation of the airships by introducing the effect of flexibility as non controlled supplementary degrees of freedom. The deformation of the blimp is not considered as a perturbation but rather acting on the motion of the airship.

The influence of structural flexibility on the dynamics of mechanical systems has become increasingly important in classical robotics $[6,7,8]$, and recently in flying robots (i.e. Airships, drones...).

\footnotetext{
* Phd student and author of correspondence, Phone: (0033) 0169477504, Fax: (0033) 0169473606, Email:Selima.Bennaceur@iup.univ-evry.fr.
} 
Several approaches, to study the problems of flexible bodies, have been proposed in the literature. These approaches can be classified into two groups. The first one uses the total Lagrangian method [9]. This consists in defining the motion relatively to a fixed reference frame, but this often leads to complex relations when describing stresses and strains in the flexible body subject to large displacements and small deformations. An Updated Lagrangian Method (U.L.M.) was proposed by Bathe \& al.[10] and developed for deformable bodies that undergo large translational and rotational displacements. The resolution of the dynamic problem is incremental. The configuration and the motion of the body are identified using a moving reference configuration representing the position of the deformable body in the preceding step. Bennaceur \& al[11] propose as reference configuration a rigid body configuration which follows the motion of the body without coinciding with it. This approach is convenient for a flying body with small deformations. The motion is given by coupled sets of rigid and elastic variables. The nonlinear equations are formulated in terms of a set of time invariant matrices expressed in a reference configuration (i.e. mass and stiffness matrices). Time-variant quantities appear in the nonlinear terms that represent the dynamic coupling between the rigid body modes and the elastic deformation. A suitable technique to actualize these terms using matrix partitioning and canonical decomposition is proposed, and the dynamic system is reduced and solved via a modal synthesis.

The Newton-Euler description [12], which is an interesting method in regard of the time computation, was extensively studied by another important group of searchers in the case of rigid airships. This choice is mainly motivated by the facility to build control or stabilization algorithms based on this model. However the use of the Newton-Euler approach in the dynamic analysis of flexible complex structures is rare[12]. We propose through this paper to extend the classical rigid bodies' model to the deformable bodies, without destroying the general formalism obtained. However this requires a total reformulation of the spatial flexible structure modelization by the use of a hybrid method based on both Lagrangian and Eulerian description. The model obtained should be acceptable for control community and closer to the one used for the rigid bodies.

On the other hand airships are also governed by the aerodynamic forces that have to be modeled. The basis to analyze the motion of a rigid body in a perfect fluid has been established in the 19th century and has been described by Lamb [13] In his work, Lamb considered the case of simple displacement in a big infinite mass of fluid where the movement of this last is entirely due to the motion of a solid, and it is irrotational and acyclic. He proved that the kinetic energy of the fluid can be expressed as a quadratic shape of the six velocities of translation and rotation of the vehicle. The derivations given by Lamb will be used in the description of the airship, in a stationary uniform atmosphere. The terms depending on the acceleration (i.e. the added masses) come from the fact that the fluid considered perfect is accelerated. When an ellipsoid body moves in an incompressible and infinite inviscid fluid in order that the external flow is everywhere irrotational and continuous, the kinetic energy of the fluid produces an effect equivalent to an important increase of the mass and of the moments of inertia of the body
[14],[15],[2]. In this paper, we introduce the added mass phenomenon in our model to have a complete dynamic model of a flying flexible object.

\section{EULERIAN DESCRIPTION OF A RIGID BODY:}

\subsection{Kinematics:}

We present an hybrid approach based first on an energetic method and using the Lagrange equations. The analysis of the motion is made with respect to an earth-fixed reference frame $R_{0}=\left(O, X_{0}, Y_{0}, Z_{0}\right)$. A parallel is then done with the Eulerian approach, and the dynamical system is expressed with the Eulerian variables.

The origin of the reference frame can be chosen in an arbitrary point such as, for example, the initial position on the ground of the airship. Its axes are selected as it follows:

$X_{0}$ : north direction

$Y_{0}:$ east direction

$Z_{0}$ : downwards direction.

We define also a second reference frame, $R_{m}=\left(G, X_{m}, Y_{m}, Z_{m}\right)$ fixed at the centre of gravity of the airship G, its axes are selected as follows:

$X_{m}$ : aligned with longitudinal axis,

$Y_{m}$ : The transverse axis,

$Z_{m}$ : The normal axis directed downwards.
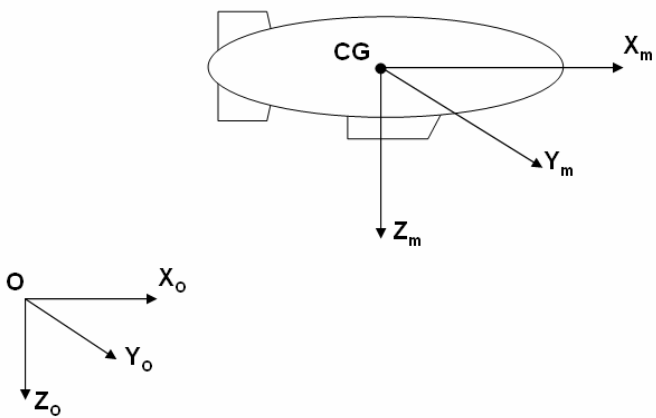

Figure 1. Definitions of the reference frames.

The airship's motion is described by the following parameters: $\eta_{1}=\left[x_{0}, y_{0}, z_{0}\right]^{T}$ : Vector position of the origin expressed in $R_{0}, \eta_{2}=[\phi, \theta, \psi]^{T}:$ The Euler angles.

The kinetic and dynamic Eulerian parameters are those used usually in aeronautics $v$ and $\dot{v}$. Where $v^{t}=\left[v_{1}^{t}, v_{2}{ }^{t}\right]$ such as: $v_{1}=[u, v, w], v_{2}=[p, q, r]$ are the linear and rotational speeds expressed in $R_{m}, \dot{v}$ are the relative acceleration expressed in $R_{m}$.

\subsection{Rotation matrices:}

Commonly in aeronautics, a parameterization in yaw, pitch and roll is used to describe the orientation of the airship in the inertial reference frame.

The configuration of the blimp is described by means of three rotations defined by three angles of orientation.

Let us note $H_{\psi}, H_{\theta}, H_{\phi}$ the orthogonal matrices of rotation. 
The whole transformation between $R_{0}$ and $R_{m}$ is the combination of these preceding matrices and is given by:

$J_{1}\left(\eta_{\mathbf{2}}\right)=\left(\begin{array}{ccc}\cos \psi \cos \theta & -\sin \psi \cos \phi+\sin \phi \cos \psi \sin \theta & \sin \phi \sin \psi+\sin \theta \cos \psi \cos \phi \\ \cos \theta \sin \psi & \cos \psi \cos \phi+\sin \theta \sin \psi \sin \phi & -\cos \psi \sin \phi+\cos \phi \sin \theta \sin \psi \\ -\sin \theta & \cos \theta \sin \phi & \cos \theta \cos \phi\end{array}\right)$

such as: $J_{1}^{T}\left(\eta_{\mathbf{2}}\right) \cdot J_{1}\left(\eta_{\mathbf{2}}\right)=J_{1}\left(\eta_{\mathbf{2}}\right) \cdot J_{1}^{T}\left(\eta_{\mathbf{2}}\right)=I_{3}$

$\mathrm{I}_{3}:$ the identity matrix.

\subsection{Transformations of velocities:}

Using the rotation matrix $J_{1}\left(\eta_{2}\right)$, the expression of the linear velocity in $R_{0}$ is given by:

$$
\dot{\eta}_{1}=J_{1}\left(\eta_{2}\right) \cdot v_{1}
$$

On the other hand, the angular velocity of the blimp $v_{2}$ is the combination of the angular velocity around the three axes of yaw, pitch and roll. It can be written related to $\dot{\eta}_{2}$ as:

$$
v_{2}=\left(\begin{array}{c}
p \\
q \\
r
\end{array}\right)=\left(\begin{array}{ccc}
1 & 0 & -\sin \theta \\
0 & \cos \phi & \sin \phi \cos \theta \\
0 & -\sin \phi & \cos \phi \cos \theta
\end{array}\right) \cdot\left(\begin{array}{c}
\dot{\phi} \\
\dot{\theta} \\
\dot{\psi}
\end{array}\right)
$$

or:

$$
\dot{\eta}_{2}=J_{2}\left(\eta_{2}\right) \cdot v_{2}
$$

The transformation matrix $J_{2}\left(\eta_{2}\right)$ is represented by:

$J_{2}\left(\eta_{2}\right)=\left(\begin{array}{ccc}1 & 0 & -\sin \theta \\ 0 & \cos \phi & \sin \phi \cos \theta \\ 0 & -\sin \phi & \cos \phi \cos \theta\end{array}\right)^{-1}=\left(\begin{array}{ccc}1 & \sin \phi \tan \theta & \cos \phi \tan \theta \\ 0 & \cos \phi & -\sin \phi \\ 0 & \frac{\sin \phi}{\cos \theta} & \frac{\cos \phi}{\cos \theta}\end{array}\right)$

It is noticed that the Euler angles parameterization have a singularity in $\theta=\frac{\pi}{2}+k \pi$.This parameterization is acceptable because it is impossible for a blimp to reach this orientation of 90 degrees pitching angle.

The global kinematics equation is then:

$$
\dot{\eta}=\left(\begin{array}{cc}
J_{1}\left(\eta_{2}\right) & 0 \\
0 & J_{2}\left(\eta_{2}\right)
\end{array}\right)\left(\begin{array}{l}
v_{1} \\
v_{2}
\end{array}\right)
$$

Notations:

Subsequently we can note $J_{1}\left(\eta_{2}\right)$ by $J_{1}$ and $J_{2}\left(\eta_{2}\right)$ by $J_{2}$.

\subsection{Dynamics:}

In order to be clearer when developing the flexible body formalism, we present here the analysis of a rigid airship by a hybrid methodology based on both Lagrangian and Euler description. The analysis of the flexible blimps is presented as an extension of the one developed for rigid flying objects.
We assumed that the airship is a rigid body with a mass $\mathrm{m}$, and $\mathrm{G}$ as centre of gravity.

The position of an arbitrary point $\mathrm{P}$ expressed in the total reference frame, of the airship is given by the following vectorial relation:

$$
\mathrm{OP}=\mathrm{OG}+\mathbf{G P}
$$

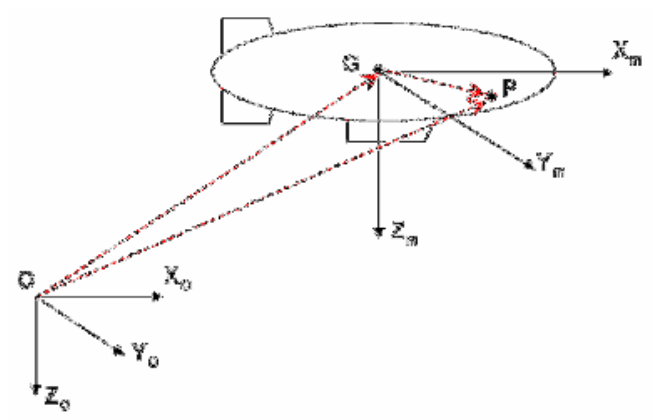

Figure 2. Definition of the position vectors.

The position of a given point $\mathrm{P}$ in the blimp is:

$$
\mathbf{q}=\eta_{1}+J_{1} \mathbf{u}_{\mathbf{0}}
$$

Let us note $\mathrm{u}_{0}$ the local position vector of the point $\mathrm{P}$ in $R_{m}$. By deriving (5) we find the following velocity's expression:

$$
\dot{\mathbf{q}}=\dot{\eta}_{\mathbf{1}}+\dot{J}_{1} \mathbf{u}_{\mathbf{0}}+J_{1} \underbrace{\dot{\mathbf{u}}_{\mathbf{0}}}_{\mathbf{0}}
$$

After computation we obtain:

$$
\begin{aligned}
\dot{J}_{1} \cdot \mathbf{u}_{\mathbf{0}} & =J_{1} \cdot\left(v_{\mathbf{2}} \wedge \mathbf{u}_{\mathbf{0}}\right)=-J_{1} \cdot\left(\mathbf{u}_{\mathbf{0}} \wedge v_{2}\right) \\
& =-J_{1} \tilde{u}_{0} v_{2}=-J_{1} \tilde{u}_{0} J_{2}{ }^{-1} \dot{\eta}_{2}
\end{aligned}
$$

$\tilde{u}_{0}$ is the skew-matrix associated to the vector $u_{0} \cdot \wedge$ is the vectorial product of two vectors. Thus the velocity vector could be expressed in a compact form as:

$$
\dot{\mathbf{q}}=\left[\begin{array}{lll}
I_{3} & J_{1} \tilde{\mathbf{u}}_{\mathbf{0}} & J_{2}^{-1}
\end{array}\right]\left(\begin{array}{l}
\dot{\eta}_{1} \\
\dot{\eta}_{2}
\end{array}\right)
$$

We will use this last form of the velocity vector thereafter in the development of the kinetic energy.

The following definition of the kinetic energy is used:

$$
E_{c}=\frac{1}{2} \int_{V} \rho \dot{\mathbf{q}}^{\mathbf{T}} \dot{\mathbf{q}} d V
$$

$\rho$ and $\mathrm{V}$ are respectively the mass density and the volume of the body. This leads to the following relation:

$$
E_{c}=\frac{1}{2} \dot{\eta}^{\mathbf{T}} M \dot{\eta}
$$

Where $\mathbf{M}$ is recognized as the symmetrical mass matrix and defined as follows: 


$$
M=\int_{V} \rho\left(\begin{array}{cc}
I_{3} & -J_{1} \tilde{\mathbf{u}}_{0} J_{2}^{-1} \\
-J_{1} \tilde{u}_{0} J_{2}^{-1} & \left(-J_{1} \tilde{\mathbf{u}}_{0} J_{2}^{-1}\right)^{T}-J_{1} \tilde{\mathbf{u}}_{0} J_{2}^{-1}
\end{array}\right) d V
$$

In a more symbolic form the mass matrix is:

$$
\begin{aligned}
& M=\left(\begin{array}{ll}
m_{T T} & m_{T R} \\
m_{R T} & m_{R R}
\end{array}\right) \text { With: } m_{T T}=\int_{V} \rho I d V \\
& m_{I R}=\int_{V} \rho\left(-J_{1} \tilde{\mathbf{u}}_{0} J_{2}^{-1}\right) d V=m_{R T} \quad, \quad m_{R R}=\int_{V} \rho\left(-J_{1} \tilde{\mathbf{u}}_{0} J_{2}^{-1}\right)^{T}\left(-J_{1} \tilde{\mathbf{u}}_{0} J_{2}^{-1}\right) d V
\end{aligned}
$$

We note that the term $m_{T T}$, associated with the translation motion, is invariant. However, the other matrices depend on the generalized co-ordinates and consequently they are implicit functions of time.

As long as the centre of gravity of the blimp coincides with the centre of the reference frame we can consider that the two extra-diagonal sub-matrices of $\mathrm{m}$ are null.

Thus:

$$
M=\left(\begin{array}{cc}
m_{T T} & 0_{3 * 3} \\
0_{3 * 3} & m_{R R}
\end{array}\right)
$$

\subsection{Lagrange's equations:}

Let us use the Lagrange's equations to define the motion of the blimp. This leads to the following relation:

$$
\frac{d}{d t}\left(\frac{\partial E_{C}}{\partial \dot{\eta}}\right)-\frac{\partial E_{C}}{\partial \eta}=\tau^{\mathrm{T}}
$$

$\tau$ represents the external forces and moments.

First of all let us evaluate the first left hand term:

$$
\frac{d}{d t}\left(\frac{\partial E_{C}}{\partial \dot{\eta}}\right)=\left[\ddot{\eta}_{1}{ }^{\mathbf{T}} m_{T T} \quad\left(\ddot{\eta}_{2}{ }^{\mathbf{T}} m_{R R}+\dot{\eta}_{2}{ }^{\mathbf{T}} \dot{m}_{R R}\right)\right]
$$

The inertia matrix $m_{R R}$ is expressed in $R_{0}$. If we note by $I_{R R}$ the constant symmetric inertia matrix in $R_{m}$, we obtain:

$$
m_{R R}=\left(J_{2}^{-1}\right)^{T} I_{R R} J_{2}^{-1}
$$

By using the following relations [16]

$$
\begin{aligned}
& \dot{J}_{2}^{-1} \dot{\eta}_{2}=0 \\
& \dot{J}_{2}^{-1} \eta_{2}=-J_{2}^{-1} \dot{\eta}_{2}
\end{aligned}
$$

and (3) : $v_{2}=J_{2}^{-1} \dot{\eta}_{2}$

and while differentiating with respect to time the equation (15), we obtain:

$$
\dot{\eta}_{2}{ }^{\mathrm{T}} \dot{m}_{R R}=v_{2}{ }^{\mathrm{T}} I_{R R} \dot{J}_{2}^{-1}
$$

When replacing the last relation in (14) it gives:

$$
\frac{d}{d t}\left(\frac{\partial E_{C}}{\partial \dot{\eta}}\right)=\left[\ddot{\eta}_{1}{ }^{\mathbf{T}} m_{T T} \quad\left(\ddot{\eta}_{2}{ }^{\mathbf{T}} m_{R R}+v_{2}{ }^{\mathbf{T}} I_{R R} \dot{J}_{2}^{-1}\right)\right]
$$

The second left term of Lagrange's equation is:

$$
\begin{aligned}
\frac{\partial E_{C}}{\partial \eta} & =\left(\begin{array}{lll}
0_{3}^{T} & \frac{1}{2} \frac{\partial}{\partial \eta_{2}}\left(\dot{\eta}_{2}{ }^{\mathrm{T}} m_{R R} \dot{\eta}_{2}\right)
\end{array}\right) \\
& =\left(\begin{array}{lll}
0_{3}^{T} & -v_{2}^{\mathbf{T}} I_{R R} & j_{2}^{-1}
\end{array}\right)
\end{aligned}
$$

$0_{3}^{T}$ is the null vector of three dimensions. The Lagrange's equation becomes then:

$$
\begin{gathered}
m_{T T} \ddot{\eta}_{1}=\tau_{1} \\
m_{R R} \ddot{\eta}_{2}=\tau_{2}-2\left(\dot{J}_{2}^{-1}\right)^{T} I_{R R} v_{2}
\end{gathered}
$$

\subsection{Eulerian description:}

In this part, we will define the motion of the blimp with the Eulerian variables starting from (21.a), (21.b) and presenting the correlation between the Lagrangian and Eulerian description.

When multiplying by $J_{2}^{-1}$ the relation (21.a), and according to the equation (15), this gives:

$$
\begin{aligned}
& J_{2}{ }^{-1}\left(J_{2}{ }^{-1}\right)^{T} I_{R R} J_{2}{ }^{-1} \ddot{\eta}_{2} \\
& =J_{2}{ }^{-1} \tau_{2}-2 J_{2}{ }^{-1}\left(J_{2}{ }^{-1}\right)^{T} I_{R R} v_{2}
\end{aligned}
$$

The following relations are proved (see for example [9]):

$$
\begin{gathered}
J_{2}{ }^{-1}\left(J_{2}{ }^{-1}\right)^{T}=4 I_{3} \\
J_{2}{ }^{-1}\left(\dot{J}_{2}{ }^{-1}\right)^{T} I_{R R} v_{2}=2 v_{2} \wedge I_{R R} v_{2}
\end{gathered}
$$

Thus, relation (22) becomes:

$$
\begin{aligned}
& 4 I_{R R} J_{2}{ }^{-1} \ddot{\eta}_{2} \\
& =J_{2}{ }^{-1} \tau_{2}-4 v_{2} \wedge I_{R R} v_{2}
\end{aligned}
$$

Using relations (3) and (16) one can easily find:

$$
\dot{v}_{2}=J_{2}^{-1} \ddot{\eta}_{2}
$$

And we obtain the dynamic relation of rotation expressed with Eulerian variables:

$$
I_{R R} \dot{v}_{\mathbf{2}}=\frac{1}{4} J_{2}^{-1} \tau_{\mathbf{2}}-v_{\mathbf{2}} \wedge I_{R R} v_{\mathbf{2}}
$$


After recalling the classical differential relation:

$$
J_{1}^{-1} \ddot{\eta}_{1}=\dot{v}_{1}+v_{2} \wedge v_{1}
$$

The dynamical system of the blimp expressed with the Euler variables becomes:

$$
\underbrace{\left(\begin{array}{cc}
m_{T T} & 0 \\
0 & I_{R R}
\end{array}\right)}_{M_{E}} \underbrace{\left(\begin{array}{l}
\dot{v}_{\mathbf{1}} \\
\dot{v}_{\mathbf{2}}
\end{array}\right)}_{\dot{v}}=\left(\begin{array}{l}
J_{1}^{-1} \tau_{\mathbf{1}}-m_{T T}\left(v_{\mathbf{2}} \wedge v_{\mathbf{1}}\right) \\
\frac{1}{4} J_{2}^{-1} \tau_{\mathbf{2}}-v_{\mathbf{2}} \wedge\left(I_{R R} v_{\mathbf{2}}\right)
\end{array}\right)
$$

Or in compact form:

$$
\mathrm{M}_{\mathrm{E}} \dot{v}=\underline{\tau}+\mathrm{Q}_{\mathrm{G}}
$$

$\mathrm{M}_{\mathrm{E}}$ is the constant mass matrix in the Eulerian description, $\underline{\tau}=\left(\begin{array}{c}\mathrm{J}_{1}^{-1} \tau_{1} \\ \frac{1}{4} \mathrm{~J}_{2}^{-1} \tau_{2}\end{array}\right)$ is the vector of external forces, and

finally $\mathrm{Q}_{\mathrm{G}}=\left(\begin{array}{l}-\mathrm{m}_{\mathrm{TT}}\left(v_{2} \wedge v_{1}\right) \\ -v_{2} \wedge\left(\mathrm{I}_{\mathrm{RR}} v_{2}\right)\end{array}\right)$ is a vector containing the gyroscopic and Coriolis forces.

\section{EULERIAN DESCRIPTION OF A FLEXIBLE BODY}

In the flexible case, the motion analysis will be similar to that used for rigid body, but with the adjunction of supplementary degrees of freedom (d.o.f). Indeed the body's flexibility will be represented by a limited number (nd) of lower frequency deformation modes, resulting from the Rayleigh-Ritz decomposition. The choice of the number of modes to be retained depends on the structure and the solicitations applied (see for example [17],[18]).

\subsection{Dynamics:}

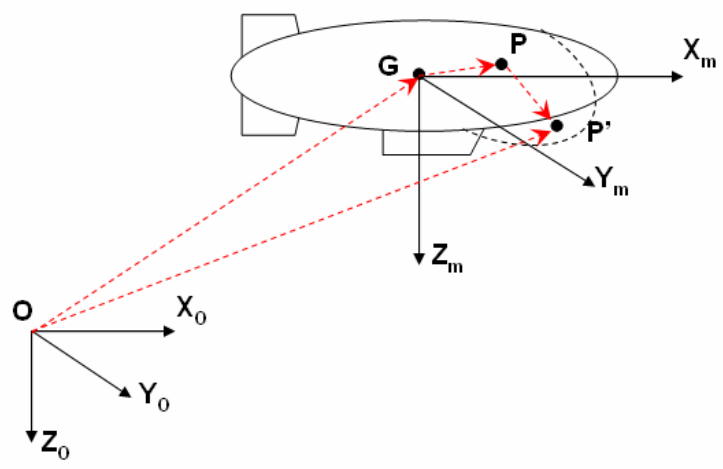

Figure 3. Definitions of the position vectors.

The position of a point in the blimp is given by: (see figure 3 )

$$
\mathrm{OP}^{\prime}=\mathbf{O G}+\mathbf{G P}+\mathbf{P P}^{\prime}
$$

Knowing that $\mathrm{P}^{\prime}$ is a point of the deformed configuration.As in the rigid case, we will start with a Lagrangian description. Consequently all the dynamic parameters are expressed in $R_{0}$. Consequently:

$$
\mathbf{q}=\eta_{1}+\mathrm{J}_{1} \underbrace{\left(\mathbf{u}_{0}+\mathbf{u}_{\mathbf{d}}\right)}_{\mathbf{U}}=\eta_{1}+\mathrm{J}_{1} \mathbf{U}
$$

By deriving the relation (32) with respect to time, and when using similar developments to those presented in (5), we obtain:

$$
\dot{\mathbf{q}}=\dot{\eta}_{1}-J_{1} \tilde{\mathbf{U}} J_{2}^{-1} \dot{\eta}_{2}+J_{1} \dot{\mathbf{u}}_{\mathbf{d}}
$$

The displacement of deformation $\mathrm{u}_{\mathrm{d}}=\mathrm{u}_{\mathrm{d}}(\mathrm{s}, \mathrm{t})$ is a function of time and space. It can be broken up into a sum of two separate functions:

$$
\mathbf{u}_{\mathbf{d}}(s, t)=\sum_{i=1}^{n d} \mathbf{Y}_{\mathbf{d}}^{\mathbf{i}}(t) X^{i}(s)
$$

Where:

$X^{i}(s)$ : represent the $\mathrm{i}^{\text {th }}$ eigenmode of the solid

$\mathbf{Y}_{\mathbf{d}}^{\mathbf{i}}(t)$ : the associated modal amplitude.

This writing can be condensed in the following way:

$$
\mathbf{u}_{\mathbf{d}}=S \overline{\mathbf{Y}}_{\mathbf{d}}
$$

$S$ represents the selected modes, and $\overline{\mathbf{Y}}_{\mathbf{d}}$ is the column matrix composed by the various $Y_{d}^{i}$.

We denote by $\bar{\eta}=\left(\begin{array}{l}\eta_{1} \\ \eta_{2} \\ \overline{\mathbf{Y}}_{\mathbf{d}}\end{array}\right)$ the total position vector of an arbitrary point of the flexible airship.

The modes of deformation $\mathrm{S}$ being constant compared to time, we can thus deduce that the speed of a given point from the blimp will be:

$$
\dot{\mathbf{q}}=\dot{\eta}_{1}-J_{1} \tilde{U} J_{2}^{-1} \dot{\eta}_{\mathbf{2}}+J_{1} S \dot{\overline{\mathbf{Y}}}_{\mathbf{d}}
$$

The preceding form of the speed will be used for the development of the kinetic energy of a deformable body.

\subsection{Expression of the kinetic energy of an arbitrary point P':}

Similarly to the developments presented in $\S 2$, we will have:

$$
E_{c}=\frac{1}{2} \dot{\bar{\eta}}^{T} M \dot{\bar{\eta}}
$$

$\mathrm{M}$ is now defined by:

$$
M=\int_{V} \rho\left(\begin{array}{c}
I \\
-J_{1} \tilde{U} J_{2}^{-1} \\
J_{1} S
\end{array}\right)\left(\begin{array}{lll}
I & -J_{1} \tilde{U} J_{2}^{-1} & J_{1} S
\end{array}\right) d V
$$

The mass matrix can be written in a symbolic form as follows: 


$$
\begin{aligned}
& M=\left(\begin{array}{ccc}
m_{T T} & m_{T R} & m_{T D} \\
m_{T R}^{T} & m_{R R} & m_{R D} \\
m_{T D}^{T} & m_{R D}^{T} & m_{D D}
\end{array}\right) \text { with: } m_{T T}=\int_{V} \rho I d V ; \\
& m_{T R}=-\int_{V} \rho J_{1} \tilde{U} J_{2}{ }^{-1} d V ; m_{D D}=\int_{V} \rho S^{T} S d V ; \\
& m_{T D}=J_{1} \int_{V} \rho S d V, m_{R R}=\int_{V} \rho\left(J_{2}{ }^{-1}\right)^{T} \tilde{U}^{T} \tilde{U} J_{2}^{-1} d V \\
& m_{R D}=\int_{V} \rho\left(J_{2}{ }^{-1}\right)^{T} \tilde{U}^{T} J_{1} d V .
\end{aligned}
$$

It is clear that the two sub-matrices respectively associated with the translation and the elastic co-ordinates are constant. The other matrices depend on the generalized co-ordinates of the system, and consequently they are implicit functions of time.

By coinciding the centre of gravity of the machine and the centre of $R_{m}$, and by assuming that the position of this last does not vary significantly in $R_{m}$ in presence of small deformations, we can thus consider that the sub-matrix $\mathrm{m}_{\mathrm{TR}}$ is null.

We will also assume here that the terms of coupling translation-deformation $\mathrm{m}_{\mathrm{TD}}$ are void.

The kinetic energy is then:

$$
\begin{aligned}
& E_{C}=\frac{1}{2}\left(\dot{\eta}_{\mathbf{1}}{ }^{\mathbf{T}} m_{T T} \dot{\eta}_{\mathbf{1}}+\dot{\eta}_{\mathbf{2}}{ }^{\mathbf{T}} m_{R R} \dot{\eta}_{\mathbf{2}}\right. \\
& \left.+2 \dot{\eta}_{\mathbf{2}}{ }^{\mathbf{T}} m_{R D} \overline{\dot{\mathbf{Y}}}_{\mathbf{d}}+\overline{\dot{\mathbf{Y}}}_{\mathbf{d}}{ }^{\mathbf{T}} m_{D D} \overline{\dot{\mathbf{Y}}}_{\mathbf{d}}\right)
\end{aligned}
$$

By using the Lagrange's equations, we obtain the following relation:

$$
M \ddot{\bar{\eta}}=\tau-K \bar{\eta}+Q_{G}
$$

Where $\tau$ is the vector of the external forces and moments, and $\mathrm{K}$ is the matrix of stiffness given by:

$$
K=\left(\begin{array}{ccc}
0 & 0 & 0 \\
0 & 0 & 0 \\
0 & 0 & K_{D D}
\end{array}\right)
$$

The relation (40) can be given in a matrix form as follows:

$$
\begin{aligned}
& \left(\begin{array}{ccc}
m_{T T} & 0 & 0 \\
0 & m_{R R} & m_{R D} \\
0 & m_{R D}^{T} & m_{D D}
\end{array}\right)\left(\begin{array}{l}
\ddot{\eta}_{\mathbf{1}} \\
\ddot{\eta}_{\mathbf{2}} \\
\ddot{\ddot{\mathbf{Y}}}_{\mathbf{d}}
\end{array}\right) \\
& +\left(\begin{array}{ccc}
0 & 0 & 0 \\
0 & 0 & 0 \\
0 & 0 & K_{D D}
\end{array}\right)\left(\begin{array}{l}
\eta_{\mathbf{1}} \\
\eta_{\mathbf{2}} \\
\overline{\mathbf{Y}}_{\mathbf{d}}
\end{array}\right)=\left(\begin{array}{c}
\tau_{\mathbf{1}} \\
\tau_{\mathbf{2}} \\
\mathbf{0}
\end{array}\right)+\left(\begin{array}{l}
Q_{G \eta_{1}} \\
Q_{G \eta_{2}} \\
Q_{G d}
\end{array}\right)
\end{aligned}
$$

Then $\quad \ddot{\bar{\eta}}=\tau-\left(\begin{array}{c}0 \\ 0 \\ K_{D D} \overline{\mathbf{Y}}_{\mathbf{d}}\end{array}\right)+Q_{G}$

Let us now analyze the vector of gyroscopic and Coriolis forces $\mathrm{Q}_{\mathrm{G}}$.

From the Lagrange's equation, we have:

$$
Q_{G}=-\dot{M} \dot{\bar{\eta}}+\left[\frac{\partial}{\partial \bar{\eta}}\left(\frac{1}{2} \dot{\bar{\eta}}^{\mathbf{T}} M \dot{\bar{\eta}}\right)\right]^{T}
$$

Or in more explicit form:

$$
Q_{G}=\left(\begin{array}{c}
Q_{G \eta_{1}} \\
Q_{G \eta_{2}} \\
Q_{G d}
\end{array}\right)=\left(\begin{array}{c}
\left.\frac{\partial}{\partial \eta_{1}}\left(\frac{1}{2} \dot{\bar{\eta}}^{\mathbf{T}} M \dot{\bar{\eta}}\right)\right]^{T} \\
-\dot{m}_{R R} \dot{\eta}_{2}-\dot{m}_{R D} \dot{\mathbf{Y}}_{\mathbf{d}}+\left[\frac{\partial}{\partial \eta_{2}}\left(\frac{1}{2} \dot{\bar{\eta}}^{\mathbf{T}} M \dot{\bar{\eta}}\right)\right]^{T} \\
-\dot{m}_{R D} \dot{\eta}_{2}-\dot{m}_{D D} \dot{\mathbf{Y}}_{\mathbf{d}}+\left[\frac{\partial}{\partial \overline{\mathbf{Y}}_{\mathbf{d}}}\left(\frac{1}{2} \dot{\bar{\eta}}^{\mathbf{T}} M \dot{\bar{\eta}}\right)\right]^{T}
\end{array}\right)
$$

The development of this relation leads to these following results:

- The first component could be written as:

$$
Q_{G \eta_{1}}=\left[\frac{\partial}{\partial \eta_{1}}\left(\frac{1}{2} \dot{\bar{\eta}}^{\mathbf{T}} M \dot{\bar{\eta}}\right)\right]^{T}=0
$$

- $\quad$ For the second component $Q_{G \eta_{2}}$ we have:

$$
Q_{G \eta_{2}}=-\dot{m}_{R R} \dot{\eta}_{2}-\dot{m}_{R D} \dot{\overline{\mathbf{Y}}}_{\mathbf{d}}+\left[\frac{\partial}{\partial \eta_{2}}\left(\frac{1}{2} \dot{\bar{\eta}}^{\mathbf{T}} M \dot{\bar{\eta}}\right)\right]^{T}
$$

The first term is:

$$
-\dot{m}_{R R} \dot{\eta}_{2}=-\overbrace{\left(J_{2}{ }^{-1}\right)^{T} I_{R R} J_{2}^{-1}} \dot{\eta}_{2}
$$

With $I_{R R}$ the matrix of rotation inertia expressed in $R_{m}$.

Basing on the fact that this analysis is intended for the airships subjected to small deformations, therefore we will keep the same assumptions used for the rigid case. Consequently the inertial terms expressed in the pointer are invariants.

\section{Notations}

We note $I_{R R}$ defined by $m_{R R}=\left(J_{2}^{-1}\right)^{T} I_{R R} J_{2}^{-1}$ the rotation matrix of the flexible body; and $I_{R D}$ defined by $m_{R D}=\left(J_{2}^{-1}\right)^{T} I_{R D} \quad$ a term of inertial coupling rotationdeformation.

Finally $I_{T D}$ defined by $m_{T D}=J_{1} I_{T D}$ is the term of inertial coupling translation-deformation. 
As announced in the beginning, we will suppose that $I_{R R}, I_{R D}, I_{T D}$ are constant matrices with respect to time through the assumption of small deformations.

And we denote $I_{T T}=m_{T T}$ and $I_{D D}=m_{D D}$ respectively the constant matrices of translation and deformation.

According to this and to the equations (16) (3) and (47) we have:

$$
-\overbrace{\left(J_{2}^{-1}\right)^{T} I_{R R} J_{2}^{-1}}^{\dot{\eta}_{2}}=-\left(\dot{J}_{2}^{-1}\right)^{T} I_{R R} v_{2}
$$

and we can say for the second term that:

$$
-\dot{m}_{R D} \overline{\dot{\mathbf{Y}}}_{\mathbf{d}}=-\left(\dot{J}_{2}^{-1}\right)^{T} I_{R D} \overline{\dot{\mathbf{Y}}}_{\mathbf{d}}
$$

For the third term:

$$
\begin{aligned}
\frac{\partial}{\partial \eta_{2}}\left(\frac{1}{2} \dot{\bar{\eta}}^{\mathbf{T}} M \dot{\bar{\eta}}\right) & =\frac{\partial}{\partial \eta_{2}}\left(\frac{1}{2} \dot{\eta}_{2}{ }^{\mathbf{T}} m_{R R} \dot{\eta}_{2}\right) \\
& =\frac{1}{2} \frac{\partial}{\partial \eta_{2}}\left(\dot{\eta}_{2}{ }^{\mathbf{T}}\left(J_{2}^{-1}\right)^{T} I_{R R} J_{2}^{-1} \dot{\eta}_{2}\right)
\end{aligned}
$$

By using the relation (16), and after differentiating, we will have:

$$
\left[\frac{\partial}{\partial \bar{\eta}_{2}}\left(\frac{1}{2} \dot{\bar{\eta}}^{\mathbf{T}} M \dot{\bar{\eta}}\right)\right]^{T}=-\left(\dot{J}_{2}^{-1}\right)^{T} I_{R R} v_{2}
$$

Thus:

$$
Q_{G \eta_{2}}=-2\left(\dot{J}_{2}^{-1}\right)^{T} I_{R R} v_{\mathbf{2}}-\left(\dot{J}_{2}^{-1}\right)^{T} I_{R D} \overline{\dot{\mathbf{Y}}}_{\mathbf{d}}
$$

- For the third component $Q_{G_{d}}$ we have:

$$
\begin{aligned}
& Q_{G d}=-\dot{m}_{R D}{ }^{T} \dot{\eta}_{2} \\
& +\underbrace{\left[\frac{\partial}{\partial \overline{\mathbf{Y}}_{\mathbf{d}}}\left(\frac{1}{2} \dot{\bar{\eta}}^{\mathbf{T}} M \dot{\bar{\eta}}\right)\right]^{T}}_{0}
\end{aligned}
$$

According to the preceding notations we can obtain the following relation:

$$
Q_{G d}=-I_{R D}{ }^{T} \underbrace{\dot{J}_{2}^{-1} \dot{\eta}_{2}}_{0}=0
$$

When collecting all these developments, the equation (41) could be expressed in this form:

$$
\begin{aligned}
& \left(\begin{array}{ccc}
I_{T T} & 0 & 0 \\
0 & m_{R R} & m_{R D} \\
0 & m_{R D}^{T} & I_{D D}
\end{array}\right)\left(\begin{array}{l}
\ddot{\bar{\eta}}_{\mathbf{1}} \\
\ddot{\bar{\eta}}_{\mathbf{2}} \\
\ddot{\overline{\mathbf{Y}}}_{\mathbf{d}}
\end{array}\right)=\left(\begin{array}{c}
\tau_{\mathbf{1}} \\
\tau_{\mathbf{2}} \\
-K_{d d} \overline{\mathbf{Y}}_{\mathbf{d}}
\end{array}\right)+ \\
& 0 \\
& \left(\begin{array}{c}
-2\left(\dot{J}_{2}^{-1}\right)^{T} I_{R R} v_{\mathbf{2}}-\left(\dot{J}_{2}^{-1}\right)^{T} I_{R D} \overline{\dot{\mathbf{Y}}}_{\mathbf{d}} \\
0
\end{array}\right)
\end{aligned}
$$

We will now transform this system equation (55) by the use of the Eulerian variables. The methodology is similar to that developed in the rigid case, and by using the relations (24), (25). This leads to the following system:

$$
\left(\begin{array}{ccc}
I_{T T} & 0 & 0 \\
0 & I_{R R} & I_{R D} \\
0 & I_{R D}^{T} & I_{D D}
\end{array}\right)\left(\begin{array}{l}
\dot{v}_{\mathbf{1}} \\
\dot{\mathrm{v}}_{\mathbf{2}} \\
\overline{\mathbf{Y}}_{\mathbf{d}}
\end{array}\right)=\left(\begin{array}{c}
J_{1}^{-1} \tau_{\mathbf{1}} \\
\frac{1}{4} J_{2}^{-1} \tau_{\mathbf{2}} \\
0
\end{array}\right)+\left(\begin{array}{c}
-I_{T T}\left(v_{\mathbf{2}} \wedge v_{\mathbf{1}}\right) \\
-v_{\mathbf{2}} \wedge\left(I_{R R} v_{\mathbf{2}}\right)-v_{\mathbf{2}} \wedge\left(I_{R D} \overline{\mathbf{Y}}_{\mathbf{d}}\right) \\
-K_{d d} \overline{\mathbf{Y}}_{\mathbf{d}}
\end{array}\right)
$$

Or in compact form

$$
\mathrm{M}_{\mathrm{E}} \overline{\dot{v}}=\underline{\tau}+\overline{\mathrm{Q}}_{\mathrm{G}}
$$

We thus obtain a Newton-Euler system equation similar to that defined in equation (30) but extended to the flexible blimps.

\section{AERODYNAMIC CONTRIBUTION:}

We show here the influence of the surrounding air on the general behavior of the flexible blimps. Let us consider a simple dynamical model for the action of the air on a body. To present this model, we assume that the flow is quasi-steady, i.e. the distribution of the velocities of particles of the medium coincides with the distribution corresponding to the steady motion of the body. Thus the medium responds only to the current motion of the body and forgets its initial conditions. Therefore, within the framework of this hypothesis, the resultant force and torque acting on the body can be represented in the form of a function of the instantaneous distribution of velocities in this body. Thus we reach at the statement of the problem of the motion of a body in a dragging medium as a problem of classical dynamics.

\subsection{Flow representation:}

To take into account the interaction of the airship with the surrounding fluid medium, a model of the flow is needed. Here, we rely on the potential flow theory corresponding to the following hypothesis:

- the air can be considered as a perfect fluid with uniform density $\rho_{\text {air }}$, i.e. an incompressible gas with vanishing viscosity,

- the flow is irrotational

Only the flow outside the airship contributes significantly to the aerodynamics. Denoting by $\mathbf{v}$ the velocity field in the fluid domain "air", the incompressibility and irrotational assumptions leads to:

$$
\nabla . \mathbf{v}=0 ; \nabla \wedge \mathbf{v}=0
$$

$\nabla$ is the gradient symbol, and the flow field may be described in terms of a potential $\mathrm{F}$ such as:

$$
\mathbf{v}=\nabla \Phi
$$

From the incompressibility constraint, it is easy to show that the potential obeys to the homogeneous Laplace equation: 


$$
\nabla^{2} \Phi=0 \quad \text { in } \Omega_{\text {air }}
$$

with Newman boundary conditions:

$$
\nabla \Phi \cdot \mathrm{n}=-\dot{\mathbf{q}} \cdot \mathrm{n} \quad \text { on the boundary }\left(\partial \mathrm{C}^{\mathrm{t}}\right)
$$

$\mathrm{n}$ is a unit vector, normal to $\left(\partial \mathrm{C}^{\mathrm{t}}\right)$ : the careen.

Thus, one of the most important characteristic of this representation is that $\mathrm{v}$ only depends on the current boundary conditions, and not on the history of the flow: the model is quasi-steady. To solve the potential equation, we use the boundary integral representation of the Laplace equation, together with standard boundary element method. It consists in the determination of a piecewise constant distribution of singularities over $\left(\partial \mathrm{C}^{\mathrm{t}}\right)$ (see [19] for details on the numerical treatment).

\subsection{Fluid forces:}

For this assumption, the pressure at any point in the fluid domain (including $\left(\partial \mathrm{C}^{\mathrm{t}}\right)$ ) is given by Bernoulli theorem:

$$
\mathrm{P}+\rho_{\text {air }}\left[\frac{1}{2} \mathbf{v} \cdot \mathbf{v}+\frac{\partial \Phi}{\partial \mathrm{t}}\right]=\mathrm{P}_{\infty}+\rho_{\text {air }} \cdot \frac{1}{2} \mathbf{v}_{\infty} \cdot \mathbf{v}_{\infty}
$$

The subscript $\infty$ denotes the undisturbed conditions far from the airship. This pressure distribution over the airship surface can be integrated to compute the resulting forces and torques. At the end, and with the linear property of the Laplace equation, the generalized fluid forces vector can be rewritten as:

$$
\mathbf{F}_{\mathbf{f}}=-\mathrm{M}_{\mathrm{ad}} \overline{\dot{v}}-\mathrm{B}_{\mathrm{f}} \bar{v}
$$

Where $M_{a d}$ is the matrix of the added masses (virtual masses), and $\mathrm{Bf}$ is a damping due to the flexibility of the hull. Taking into account the previous developments, the dynamic equation (57) becomes:

$$
\mathrm{M}_{\mathrm{E}}^{\prime} \overline{\dot{v}}=\underline{\tau}+\overline{\mathrm{Q}}_{\mathrm{G}}^{\prime}
$$

we note $\mathrm{M}_{\mathrm{E}}^{\prime}=\mathrm{M}_{\mathrm{E}}+\mathrm{M}_{\mathrm{ad}}, \overline{\mathrm{Q}}_{\mathrm{G}}^{\prime}=\overline{\mathrm{Q}}_{\mathrm{G}}-\mathrm{B}_{\mathrm{f}} v$. The effect of the fluid on the structure is then represented mainly by the adjunction of the added masses matrix Mad to the mass matrix of the structure.

For a quasi-ellipsoid airship the extra-diagonal terms of $\mathrm{M}_{\mathrm{ad}}$ can be neglected (for more details about the constitutive terms of $M_{a d}$, the reader can see [2]'[13]).

\section{SIMULATIONS RESULTS:}

The method for computation is based on the following flow chart:

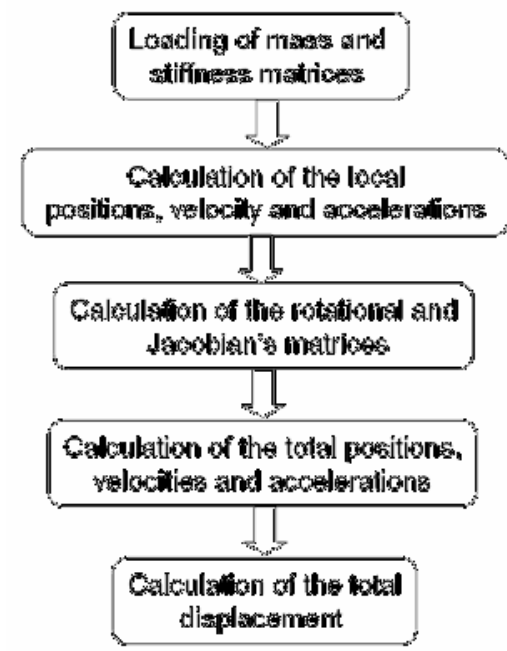

To illustrate this Eulerian approach we study the blimp belonging to the LSC-IBISC and having the following characteristics:

-The envelope:

Length: $6.25 \mathrm{~m}$.

Diameter: $1.52 \mathrm{~m}$.

Volume: $7.48 \mathrm{~m} 3$.

-Mass of the airship: $5.8 \mathrm{Kg}$.

-Payload: $1.58 \mathrm{Kg}$.

The blimp is thrust by two contrarotating propellers.

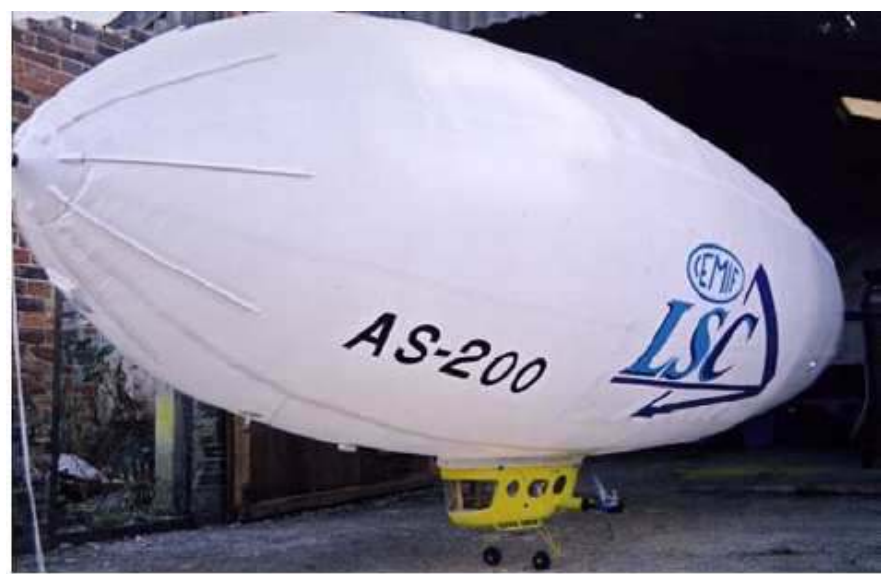

Figure 4. The LSC-IBISC's airship.

In the first step we apply to the blimp two opposite forces in the propellers to generate a yaw motion (Fig 5,6). The flexible airship should rotate about $90^{\circ}$ around the z-axis. A Proportional-Derivative (P.D) controller will impose this task, and we study the behavior of the airship during this maneuver. The number of deformable modes kept is $n d=2$. This number seems to give an acceptable approximation of the flexible behavior. We were guided for that by the modal masses of these two modes which represent roughly $70 \%$ of the total mass of the airship.

We applied the force on the point $\mathrm{P}(2,0,0)$ of the blimp.

In this simulation we applied around the yaw angle a torque consigns : 
$f_{e g}=\left(\begin{array}{c}0 \\ 0 \\ 0 \\ 0 \\ 0 \\ k_{d}\left(\psi_{d}-\psi\right)+k_{v}\left(\dot{\psi}_{d}-\dot{\psi}\right)\end{array}\right)$

With $\mathrm{K}_{\mathrm{d}}=800, \mathrm{~K}_{\mathrm{v}}=50, \psi_{\mathrm{d}}$ : The desired angular position and $\psi$ : The angular position of the airship.

One notices in figure 5 and 6 that the amplitude of the deviation of the yaw angle decreases significantly in a few seconds. it stabilizes itself while merging with the instruction.

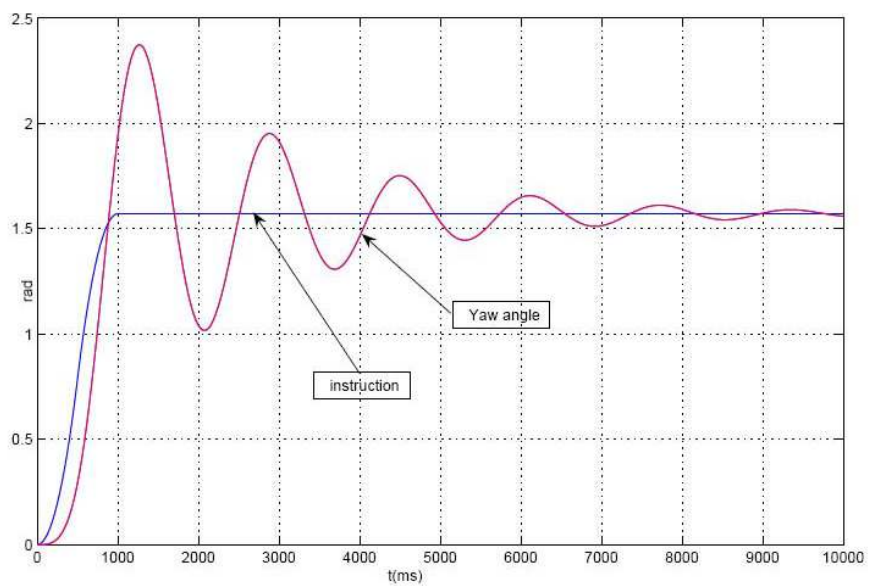

Figure 5. Superposition of the deviation of the yaw angle and the instruction.
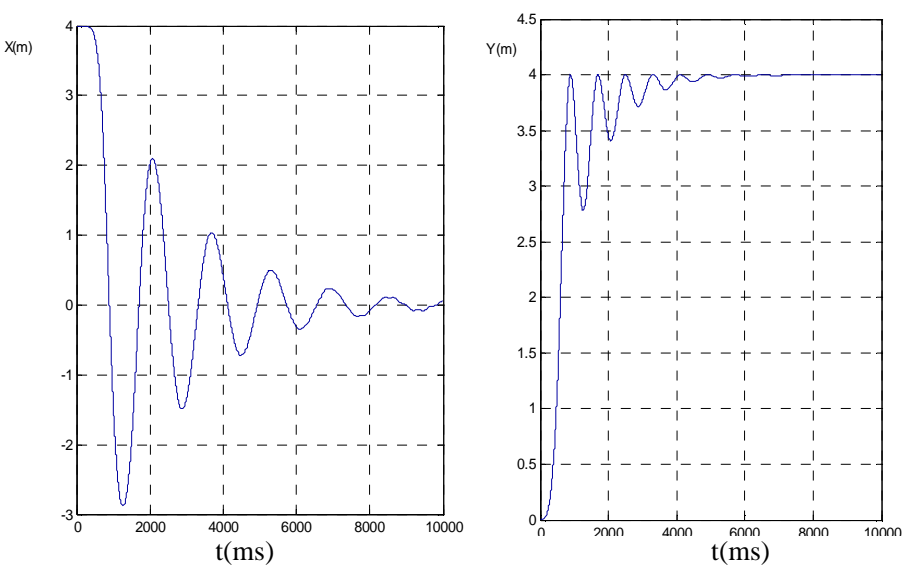

Figure 6. Position of the tip of the airship.

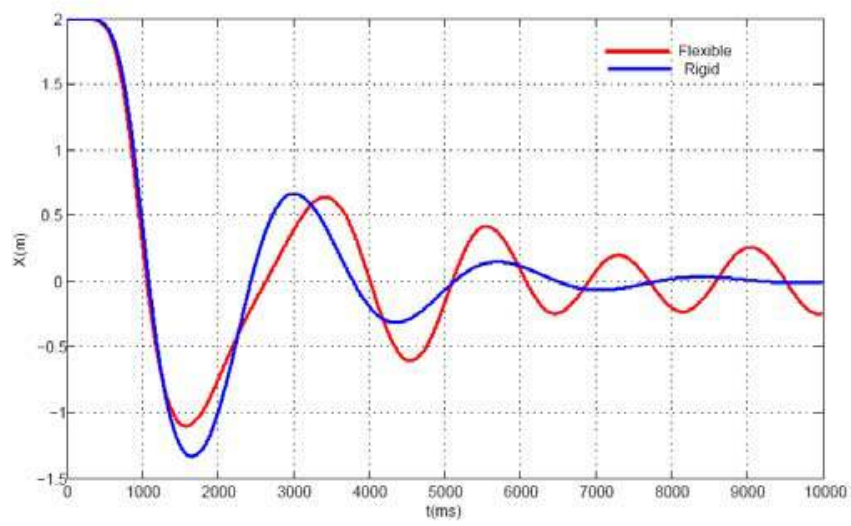

Figure 7. Superposition of the motions of the flexible and rigid airship.
In figure 7 we superimpose total displacement along the $\mathrm{X}$ axis of the rigid airship and the flexible device. It is noticed that the flexible device continues to oscillate which proves the impact of flexibility on displacement. For the rigid behavior we eliminate the two deformable modes and keep only the rigid motion.

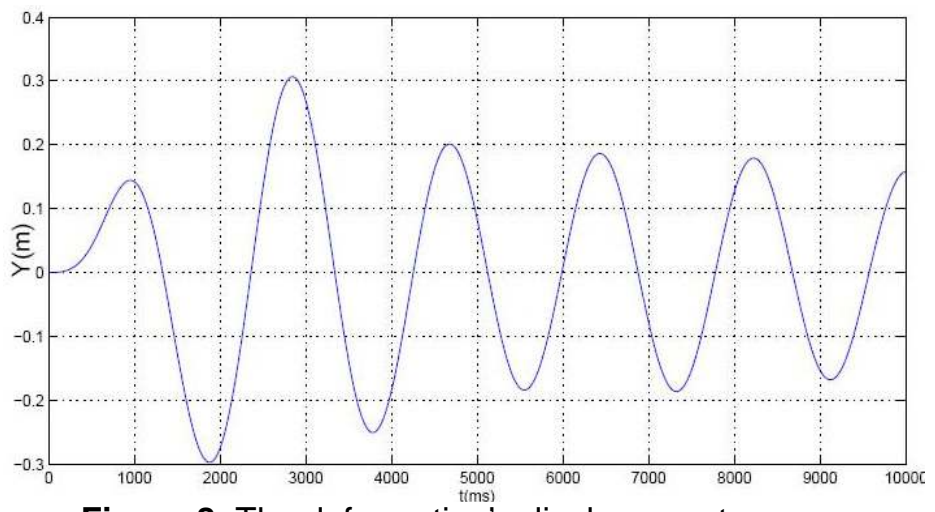

Figure 8. The deformation's displacement.

The deformations (in figure 8 ) are about $0.20 \mathrm{~m}$ what is more or less significant considering one has small deformations. In this simulation we visualized the prow of the airship, and we see well the oscillation of this later with non-negligible amplitude.

One classical motion of an airship is the helicoidally motion. This usually represents the optimal trim trajectories.

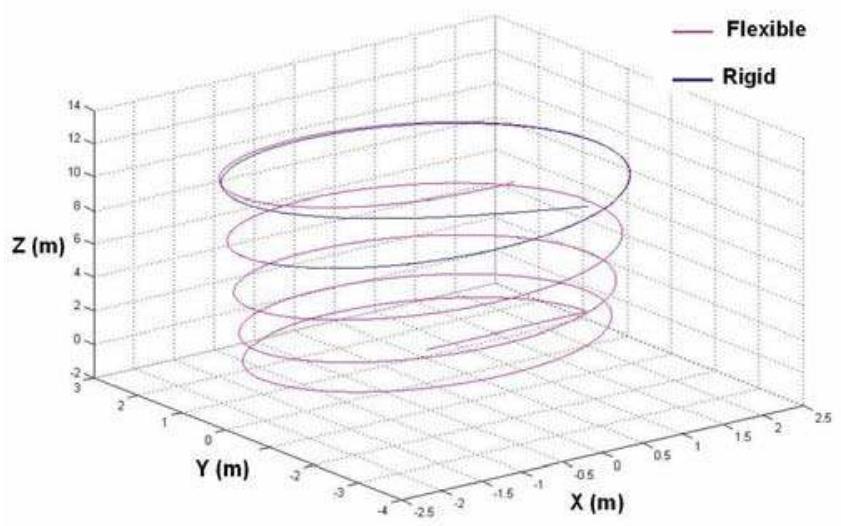

Figure 9. Rise of the airship.

This represents a complete aerial motion of the airship. The propellers were oriented adequately to assure a combined motion along the moving $\mathrm{x}$-axis and $\mathrm{z}$-axis and the tails were oriented to give a homogeneous yaw motion. We can see (in figure 9) the influence of the deformation on the overall motion of the airship. This proves that in our model, the deformation is not a sample perturbation around the main rigid motion, but it acts on this motion. Experimental data will be available in few months to validate the results of our scheme. Finally we show the influence of the added masses on the total displacement of the airship. 


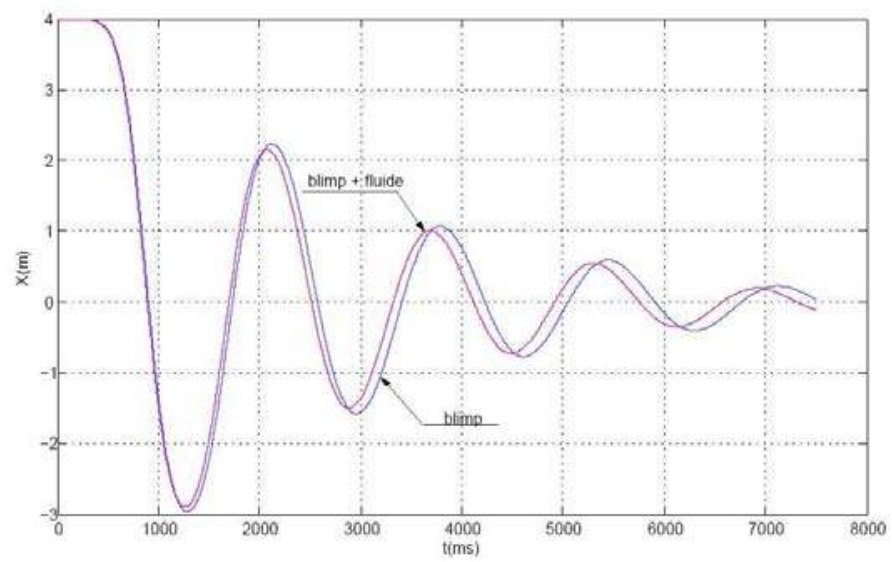

Figure 10. The impact of the fluid on the Blimp.

It is noticed (in figure 10) that the immersed airship has a delay compared to the body alone and this is because of the addition of the virtual masses representing the influence of the displacement of the mass of air around the airship when this last accelerates.

\section{CONCLUSION}

In this paper we presented a description of the flexible airships by an Eulerian method. We introduced the flexibility of the airship as an extension of a rigid body algorithm based on Eulerian description and taking into account the coupling between the rigid body motion and the deformation. A hybrid methodology is used, based on both Lagrangian and Eulerian theory. The final goal of developing this model is to enable an easy implementation of control and stabilization algorithms for the flexible flying objects. The model built presents also an interesting ratio precision/computational time. Simulation results prove that the integration of the flexibility in the dynamic system of the airship is very important and could not be neglected. The added mass phenomenon is also taken into account. However we neglected in this study the added masses issued from the vibration of the airship in the air and their coupling with those issued from the overall rigid body motion. This point is under investigation and will be presented in future works.

\section{REFERENCES}

[1] Khoury G.A.,Gillet J.D.,1999 "Airship Technology", Cambridge Univ. Press.

[2] Fossen T., 1996. "Guidance and control of ocean vehicles", J. Wiley press, Chichester,

[3] Bestaoui Y.,Hamel T. 2000 "Dynamic modeling of small autonomous blimps" Proc. Of Conference on Methods and Modeles in Automation and Robotics (MMAR00), Miedzyzdroje, Poland,pp.579-584.
[4] Calise A.,Nakwan K., 2002 " Adaptive compensation for flexible dynamics". AIAA Guidance, navigation and control conference. Montery, Canada.

[5] Bianchin M.,Quaranta G.,Mantegazza P., 2003 "State space reduced order models for static aeroelasticity and flight mechanics of flexible aircrafts". $17^{\text {th }}$ national conference AIDAA,Italy,

[6] Simo J.C., 1987 "The role of non-linear theories in transient dynamic analysis of flexible aircrafts», Journal of Sound \&Vibration, 119, pp. 487-508.

[7] Pascal. M. 1991, "Vibrations analysis of flexible multibody systems", Dyn. \& Stability of systems, Vol.6, $\mathrm{N}^{\circ} 3$.

[8] SchiehlenW, Cuse N. and Seifried R.,2006 "Multibody dynamics in computational mechanics and engineering applications", Computer Methods in Applied Mechanics and Engineering, Volume 195, Issues 41-43, 15 August 2006, pp. 5509-5522.

[9] Shabana A., 1988 "Dynamics of multibody systems". Edition Springer-Verlag.

[10] Bathe K.J \& al. 1975 "Finite Elements for large deformation dynamic analysis" INT.Jour. For Num. Meth In Eng., Vol 9, pp.353-386.

[11] Bennaceur S., Azouz N., Boukraa D. 2006 "An efficient modelling of flexible Airships: Lagrangian approach". Proceeding of the ESDA'06 ASME International Conference. ESDA2006-95741. Torino, Italy, July .

[12] Boyer F., Coiffet Ph. 1996 "Generalization of NewtonEuler model for flexible manipulators", Int. Jour. For Robotics Research 17(3), pp. 282- 293.

[13] Lamb H., 1945 "On the motion of solids through a liquid. Hydrodynamics", Dover, New York, $6^{\text {th }}$ edition.

[14] Thomasson P.G 2000 "Equation of motion of a vehicle in a moving fluid", Jour. of Aircraft, Vol.37, $\mathrm{N}^{\circ} 4$, pp. 630-639.

[15] Ortega J.,Rosier L.,Takahashi T 2006 “ On the motion of a rigid body immersed in a bidimensional incompressible perfect fluid", Annales de l'institut Poincare ( c ) Non linear Analysis .

[16] Goldestein 2001 "Classical Mechanics", Ed. Addison Wesley Publishing Company; Boston, $3^{\text {rd }}$ edition.

[17] Gibert R-J "Vibrations des structures. Interactions avec les fluides", Ed. Eyrolles, Paris, 1988, pp. 137-140.

[18] Clough R-W., PENZIEN J., 1993 "Dynamics of Structures", McGraw; International edition.

[19] Katz J., Plotkins A. 1991 "Low-speed Aerodynamics, from Wings Theory to Panel Methods", McGraw-Hill Book Co, New York. 